\title{
Análisis de las propiedades mecánicas del compuesto de matriz poliéster reforzado con fibra de vidrio 375 y cabuya aplicado a la industria automotriz
}

\section{(Analysis of the mechanical properties of the composite of polyester matrix reinforced with glass fiber 375 and cabuya applied to the automotive industry)}

\author{
Juan G. Paredes Salinas ${ }^{1}$, Cristian F. Pérez Salinas ${ }^{1}$, Christian B. Castro Miniguano ${ }^{1}$
}

\begin{abstract}
Resumen:
Los estudios de los materiales compuestos juegan un papel importante en aplicaciones de ingeniería, materiales, metalurgia y mecánica. Las fibras reforzadas con polímeros son ampliamente utilizadas en la industria automotriz e industria aeronáutica debido a sus beneficios como bajo costo, control de ruido, bajo peso y facilidad de procesamiento. El objetivo de esta investigación fue preparar un compuesto a base de fibra de vidrio 375 (FV) con adiciones de fibra natural de cabuya ( $F C$ ) en estratificaciones de fibra natural corta de cabuya (FCO1-30\%) y fibra larga del mismo (FL-30\%). Los resultados muestran un mejor comportamiento mecánico a tracción en un $7,7 \%$ respecto al material comúnmente utilizado. Se observó que la fibra larga al $30 \%$ en un orden de capas, $\mathrm{FV}+\mathrm{FC}+\mathrm{FV}$, es un potencial refuerzo del material hibrido alternativo para aplicaciones automotrices. Además, se evidenció una equilibrada organización de refuerzos, $\mathrm{FV}+\mathrm{FC}$, y adherencia microestructural con la matriz de refuerzo polimérico (RP) mediante microscopía de barrido. Los resultados del esfuerzo a la tracción y la deformación axial de la mejor combinación del material compuesto, $\mathrm{FL}-30 \%$, son validados a través del método de elementos finitos (MEF).
\end{abstract}

Palabras clave: cabuya; esfuerzos; tracción; materiales híbridos; automotriz.

\begin{abstract}
:
Studies of composite materials play an important role in engineering, materials, metallurgy and mechanical applications. Polymer reinforced fibers are widely used in the automotive and aeronautical industry because of their benefits such as low cost, noise control, low weight and ease of processing. The objective of this research was to prepare a composite based on 375 (FV) glass fiber with additions of natural cabuya (CF) fiber in laminations of short natural fiber of cabuya (FCO1-30\%) and long fiber of the same (FL-30\%). The results showed a better mechanical tensile behavior in $7.7 \%$ compared to the material commonly used. It was observed that $30 \%$ long fiber in a layer order, $\mathrm{FV}+\mathrm{FC}+\mathrm{FV}$, is a potential reinforcement of the alternative hybrid material for automotive applications. In addition, a balanced reinforcement organization, $\mathrm{FV}+\mathrm{FC}$, and micro structural adhesion with the polymeric reinforcement matrix (RP) were evidenced by scanning microscopy. The results of tensile stress and axial deformation of the best combination of composite material, FL-30\%, are validated using the finite element method (MEF).
\end{abstract}

Keywords: cabuya; stress; traction; hybrid materials; automotive.

\footnotetext{
${ }^{1}$ Universidad Técnica de Ambato, Ambato - Ecuador ( \{jgparedes, cf.perez, cb.castro\} @uta.edu.edu.ec )
} 


\section{Introducción}

El mercado mundial de los materiales compuestos (materiales ligeros) utilizados en el transporte ha tenido un crecimiento importante en los últimos años. Estos materiales alcanzaron valores por comercialización y manufactura de 115,400 millones de dólares en 2014 y se espera que este mercado alcance unos 188,700 millones de dólares en 2020, registrando una tasa de crecimiento anual compuesta del 11.6\% de 2015 a 2020 (McWilliams, 2015). Potenciales aplicaciones están teniendo los compuestos híbridos globalmente, sobre todo en la industria automotriz. Dentro del campo automotriz se encuentra la manufactura del autobús, la cual se encuentra en constante crecimiento en América Latina (Casanova, 2010). La fibra de vidrio ha incrementado su uso en los últimos 15 años. Este material de matriz polimérica reforzada con fibras sintéticas han incrementado su aplicación en varios componentes de forrado exterior e interior de productos tales como: techos, frentes, respaldos, tableros, consolas, entre otros. Pero se está experimentando la inclusión de biocompuestos para reducir el impacto ambiental en dicha industria.

Los compuestos unidireccionales y los compuestos laminados son los materiales compuestos más utilizados con una dominación de las fibras de vidrio (Hallal, Elmarakbi, Shaito, \& El-Hage, 2013). En comparación con materiales tradicionales, los materiales compuestos presentan favorables propiedades físico-mecánicas como: alta resistencia específica a la tracción y compresión, conductividad eléctrica controlable, bajo coeficiente térmico, buena resistencia a la fatiga e idoneidad para la producción de objetos de forma compleja (Pickering, Efendy, \& Le, 2016). Muchas de estas propiedades y sobre todo la capacidad y facilidad de conformación, le otorgan a este tipo de material compuesto una gran ventaja comparado con materiales convencionales como el acero y la madera. Por estas razones estos materiales son adecuados en aplicaciones de la industria automotriz, producción de energía eólica, industria náutica y aeronáutica. Las últimas tendencias en materiales compuestos son el surgimiento de polímeros reforzados con fibras naturales, compuestos verdes, como remplazo de polímeros reforzados con fibra de vidrio; esto reduce el impacto ambiental por su baja tasa de degradación (Chiaberge, 2011). Los cultivos no alimentarios y otros recursos biorenovables ofrecen una oferta casi ilimitada de materias primas renovables y potencialmente sostenibles para la producción de biocompuestos (Fowler, Hughes, \& Elias, 2006). Por esta razón, las industrias manufactureras de índole estructural y automotriz buscan la aplicación de materiales amigables con el ambiente con propiedades mecánicas similares o mejores que satisfagan los esfuerzos sometidos a sus productos finales.

Típicamente, un compuesto artificial consiste en una fase de refuerzo de material fuerte, con frecuencia de naturaleza fibrosa, incrustado en una fase de matriz continua. Este último es a menudo más débil y más conforme que el anterior. Dos de las funciones principales de la matriz son transmitir cargas aplicadas externamente al refuerzo y protegerlas de daños ambientales y mecánicos a través de tensiones de cizalla en la interfaz (Shaharuddin \& Matthews, 1994). La ventaja de tal acoplamiento es la alta resistencia y rigidez de las fibras que en la mayoría de las 
situaciones prácticas no pueden transmitir cargas. Los compuestos pueden ser clasificados de acuerdo con el tipo de matriz; de ahí que, existen compuestos de matriz metálica, cerámica y polimérica. La aplicación final a la que se va a destinar el material compuesto dicta la matriz que se debe escoger. La mayoría de compuestos poliméricos tradicionales emplean matrices termoestables (Chung, 2010). Estas resinas termoestables son líquidos viscosos que por efecto de una reacción de endurecimiento forman una gran red molecular integrada por una serie de enlaces cruzados entre cadenas moleculares que dan forma a un sólido que no puede ser reprocesado. El curado se da por el desprendimiento de calor interno (reacción exotérmica) o por calor inducido, Figura 1. Estas resinas son normalmente isotrópicas, es decir que las propiedades son idénticas en todas las direcciones. Su propiedad más característica es su respuesta al calor ya que no se funden al calentarlas. Sin embargo, su rigidez decrece a la temperatura de distorsión térmica, la cual precisa un límite superior efectivo determinante a la hora de su utilización en componentes estructurales. Esta característica es una desventaja puesto que los polímeros termoestables no pueden ser reciclados o reutilizados, ya que han sufrido modificaciones en su estructura química a nivel molecular (Hull, 2003; Smith et al., 2006).

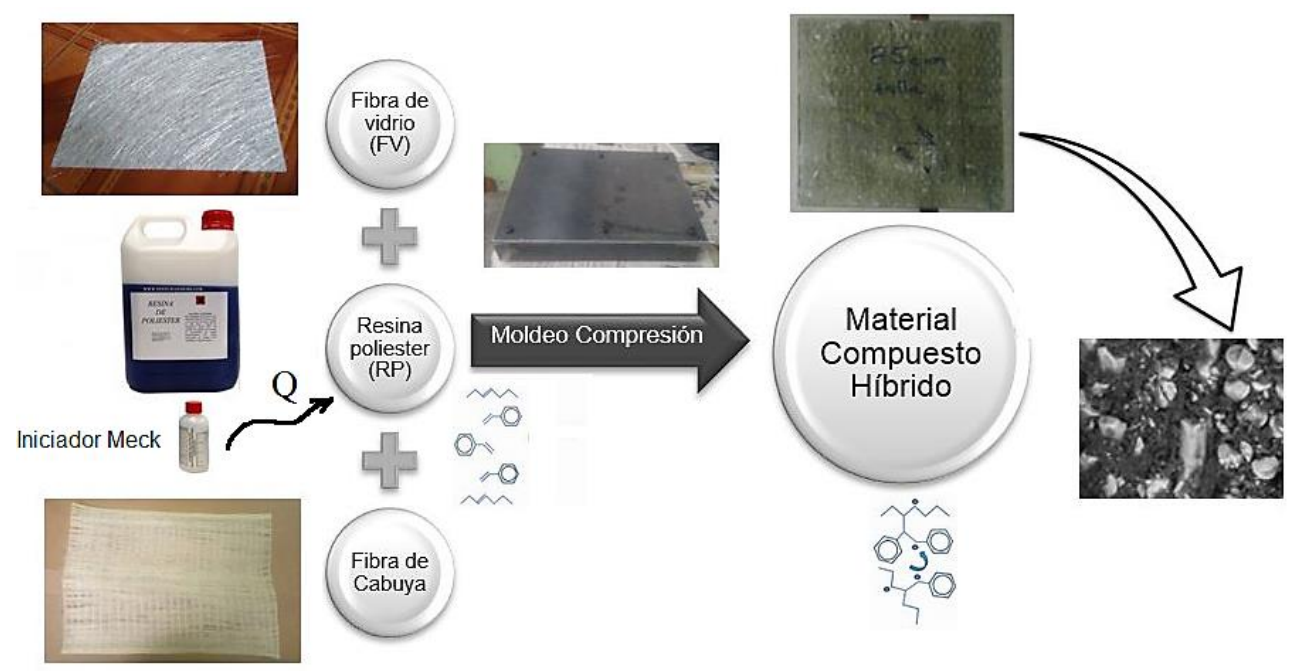

Figura 1. Proceso de elaboración de material compuesto híbrido

Estudios anteriores han encontrado los efectos de la fracción volumétrica y la longitud de las fibras naturales sobre las propiedades mecánicas de materiales compuestos biodegradables (Shibata, Cao, \& Fukumoto, 2005); inclusive, las propiedades mecánicas de los compuestos verdes fabricados a partir de fibras tratadas con álcali fueron superiores a las fibras no tratadas (Cao, Shibata, \& Fukumoto, 2006). Además, se observó en otros estudios el aumento en el rendimiento de materiales híbridos no solo en sus propiedades sino también en la adhesión de la fibra-matriz (Dieu, Phai, Ngoc, Tung, \& Le Phuong, 2004). Por todo lo anteriormente mencionado, el presente trabajo pretende determinar las propiedades fundamentales del compuesto híbrido de fibra de vidrio y cabuya mediante el proceso de estratificación. Adicionalmente, este estudio busca determinar combinaciones en fracciones volumétricas correctas con el fin de obtener las mejores propiedades mecánicas con miras a aplicaciones en la industria automotriz y otras. Finalmente se propone 
corroborar un ensayo de tracción simulada bajo un software de elementos finitos para comparar con los resultados de las pruebas experimentales bajo las mismas condiciones normativas.

\section{Materiales y metodología}

\subsection{Materiales}

Todos los materiales que se emplearon para la elaboración de probetas de material compuesto; se describen en la Tabla 1. Donde se detallan la marca, característica relevante y su procedencia.

La fibra natural (FC) crece y se cultiva naturalmente en la localidad de Salasaca perteneciente a la provincia de Tungurahua, Ecuador, que se adhiere como refuerzo para formar un compuesto híbrido. La fibra de cabuya (gabazo) que se obtiene de la hoja de la cabuya (Furcraea Andina), planta silvestre que crece y se cultivada en casi todos los sectores rurales de la sierra del Ecuador como material de amarre; dicha planta arrocetada se desarrolla hasta alcanzar una longitud promedio de hasta 1.5 metros de altura en su punto de maduración. Para el análisis del compuesto híbrido se emplearon las siguientes configuraciones: fibra corta de $10 \mathrm{~mm}$, fibra continua y tejido plano.

Tabla 1. Materiales empleados para la fabricación de probetas de material compuesto

\begin{tabular}{cccc}
\hline Material & Marca & Característica & País de origen \\
Fibra de vidrio & Jushi & $\begin{array}{c}\text { Fibra sintética de tela no } \\
\text { tejida de } 375 \mathrm{gr} / \mathrm{m}^{2}\end{array}$ & China continental \\
Fibra de cabuya & - & Fibra natural tipo gabazo & Tungurahua, Ecuador \\
Resina poliéster & Andercol 836 & Resina no insaturada & Medellín, Colombia \\
Octoato de cobalto & Andercol & al 12\% p/p & Colombia \\
Peróxido de metil-etil cetona & - & Iniciador de polimericación & Ecuador \\
Cera desmoldante & Simoniz & Cera parafínica en pasta & Colombia
\end{tabular}

Como matriz termoestable se empleó resina poliéster insaturada, que a su vez fue diluida con estireno monómero. Para iniciar la reacción de solidificación o curado de la resina se empleó el componente octoato de cobalto al $12 \%$ p/p y catalizador peróxido de metil-etil cetona (MEKP) y para facilidad de extracción de las piezas de la matriz, se empleó cera desmoldante.

En la caracterización del material mediante probetas normalizadas se utilizó la máquina universal de ensayos marca Tinius Olsen modelo H25KS con capacidad de $25 \mathrm{KN}$ en su doble columna, resolución $0.001 \mathrm{~mm} / \mathrm{min}$ para velocidad y $0.001 \mathrm{~mm}$ en desplazamiento; facilitado por la Escuela Politécnica Nacional y una máquina para ensayos de impacto por caída de dardo con sujeción de probetas con 2 actuadores neumáticos de $50 \mathrm{~mm}$ de diámetro y los anillos fijo y móvil; y un dardo de $2000 \mathrm{~g}$ liberado desde $150 \mathrm{~cm}$ de altura. Para la estratificación del material se fabricaron moldes de acero AISI A-36 macho-hembra con la configuración geométrica de las probetas, y una refinación de medidas normalizadas por proceso de cizalladura. 
Se empleó con fines comparativos una simulación dinámica FEM de test de tracción utilizando el software ANSYS SpaceCLAIM. Finalmente, para la determinación de la adherencia y homogeneidad de la matriz respecto a los refuerzo, se utilizó la técnica de microscopía de barrido SEM de marca VEGA 3 TESCAN.

\subsection{Metodología}

En primera instancia se manufacturaron moldes en acero para la obtención de probetas (Figura 2) en las distintas combinaciones de refuerzos del compuesto para efectuar ensayos de tracción acorde a la normas ASTM D3039-08 (D3039 ASTM, 2008). Posteriormente, se realizaron pruebas complementarias de flexión e impacto para corroborar resultados bajo normas ASTM D7264M-07 (D7264 ASTM, 2007) y ASTM D5628-10 (D5628 ASTM, 2010), respectivamente. La obtención de probetas se realizó mediante la técnica de estratificación por compresión mediante un cierre mecánico de moldes por presión mecánica atornillada. La resina poliéster fue diluida con estireno monómero más la adición de octoato de cobalto y peróxido de metil etil cetona sobre la resina poliéster. Las fibras de refuerzo fueron humedecidas por inmersión en la solución, para posteriormente ser colocados sobre la superficie de los moldes previamente limpiadas y recubiertas con cera desmoldante.

( a )
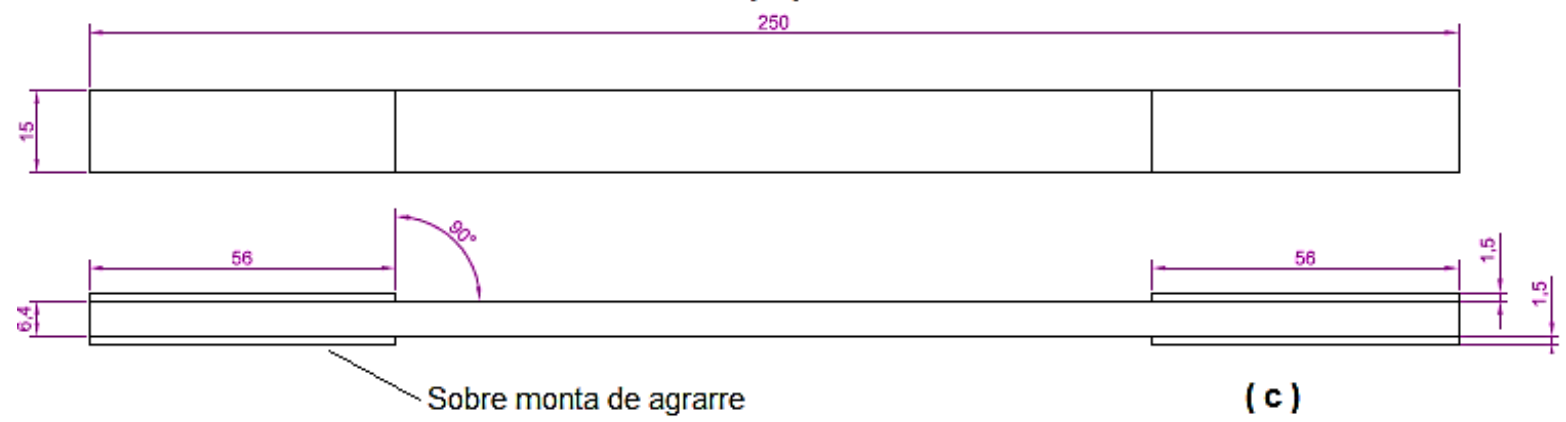

(b)

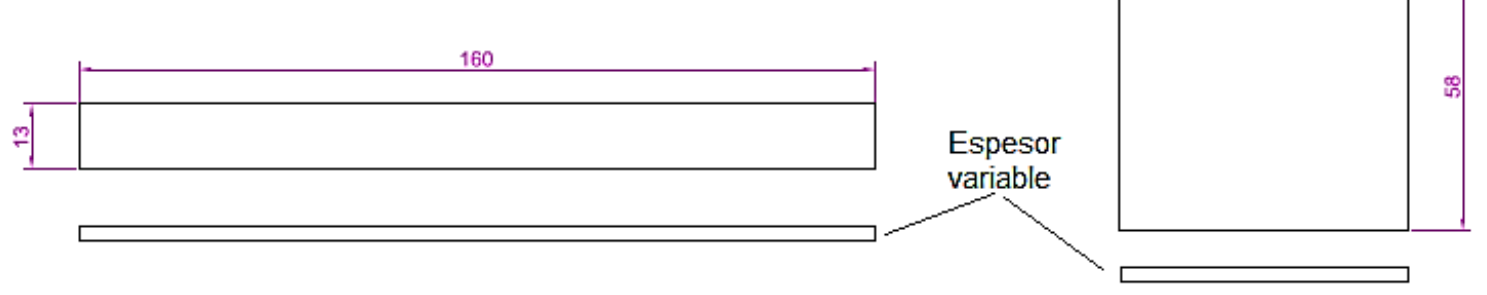

Figura 2. Dimensiones de probetas para ensayos: (a) tracción, (b) flexión, (c) impacto

Se realizaron ensayos preliminares de tracción con las estratificaciones tradicionales $(F V+F C)$ típicamente usados por los tres fabricantes de carrocerías en Tungurahua a velocidad de ensayo de 2 mm/min (D3039 ASTM, 2008). Luego, se ejecutó una nueva estratificación con componentes híbridos $(\mathrm{FV}+\mathrm{FC}+\mathrm{FV})$ con el fin de comparar resultados de propiedades mecánicas. Las combinaciones para la ejecución de ensayos se presentan en la Tabla 2. 
Tabla 2. Configuración y fracciones volumétricas del hibrido propuesto (25\% - 30\% - 40\%).

\begin{tabular}{|c|c|c|c|c|c|c|}
\hline$\#$ & $\begin{array}{l}\text { CONFIGURACIÓN } \\
\text { DEL REFUERZO }\end{array}$ & $\begin{array}{l}\text { \% REF } \\
\text { APROX. }\end{array}$ & $\begin{array}{l}\text { \# DE CAPAS } \\
\text { DE F.V - } 375\end{array}$ & $\begin{array}{c}\text { \# DE CAPAS } \\
\text { DE F.C }\end{array}$ & $\begin{array}{l}\text { ORDEN DE } \\
\text { CAPAS }\end{array}$ & $\begin{array}{c}\text { \# DE } \\
\text { PROBETAS }\end{array}$ \\
\hline \multirow[t]{4}{*}{2} & & $25 \%$ & $1(10 \%)$ & $1(15 \%)$ & FV-FC & 7 \\
\hline & \multirow{3}{*}{$\begin{array}{l}\text { FIBRA CORTA } \\
\quad(10 \mathrm{~mm})\end{array}$} & \multirow{2}{*}{$30 \%$} & $1(10 \%)$ & $2(20 \%)$ & FV-FC-FC & 7 \\
\hline & & & $2(20 \%)$ & $1(10 \%)$ & FV-FC-FV & 7 \\
\hline & & $40 \%$ & $2(20 \%)$ & $1(20 \%)$ & FV-FC-FV & 7 \\
\hline \multirow[t]{3}{*}{3} & & $25 \%$ & $1(10 \%)$ & $1(15 \%)$ & FV-FC & 7 \\
\hline & FIBRA LARGA & $30 \%$ & $2(20 \%)$ & $1(12 \%)$ & FV-FC-FV & 7 \\
\hline & & $40 \%$ & $2(20 \%)$ & $2(20 \%)$ & FV-FC-FC-FV & 7 \\
\hline \multirow[t]{4}{*}{4} & & $25 \%$ & $1(10 \%)$ & $1(15 \%)$ & FV-FC & 7 \\
\hline & TEJIDO PLANO & $30 \%$ & $2(20 \%)$ & $1(12 \%)$ & FV-FC-FV & 7 \\
\hline & & $40 \%$ & $2(20 \%)$ & $1(20 \%)$ & FV-FC-FV & 7 \\
\hline & \multicolumn{5}{|c|}{ Número Total de Probetas } & 77 \\
\hline
\end{tabular}

En la caracterización para la obtención de la deflexión y el esfuerzo máximo a flexión (Figura 3) se empleó la misma máquina Tinius Olsen modelo H25KS ya mencionada con la ayuda de aditamentos propios del ensayo de flexión concordante a norma ASTM D 7264-07. Paralelamente, con la especificación dimensional de las probetas que fueron de $160 \mathrm{~mm}$ de largo, $13 \mathrm{~mm}$ de ancho y espesor de $3.2 \mathrm{~mm}$. Para la determinación de la separación de puntos de apoyo del ensayo resultante en $102.4 \mathrm{~mm}$ concomitante al procedimiento dictado por (D7264 ASTM, 2007), que corresponde a 32 veces el espesor. La cantidad determinada previamente de probetas fue mínima de siete y velocidad de aplicación de la carga para el ensayo que fue de $1 \mathrm{~mm} / \mathrm{min}$.

(a)

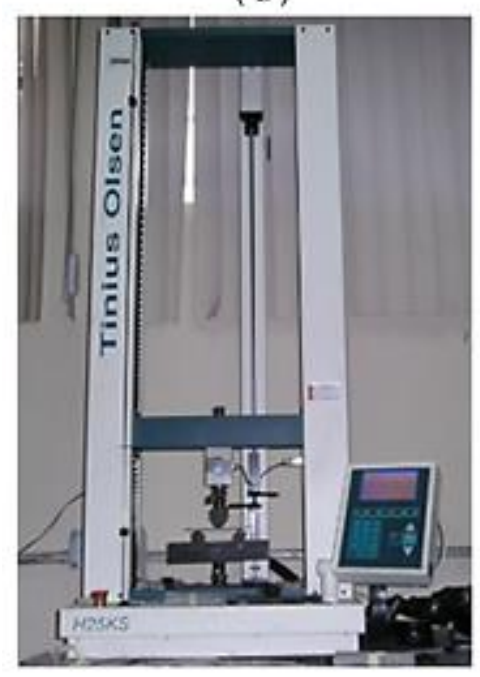

(b)
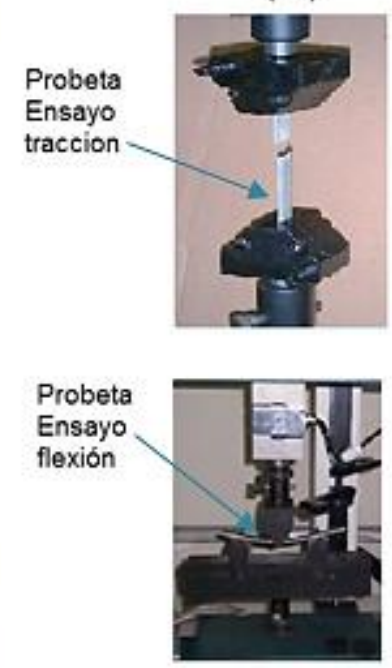

(c)

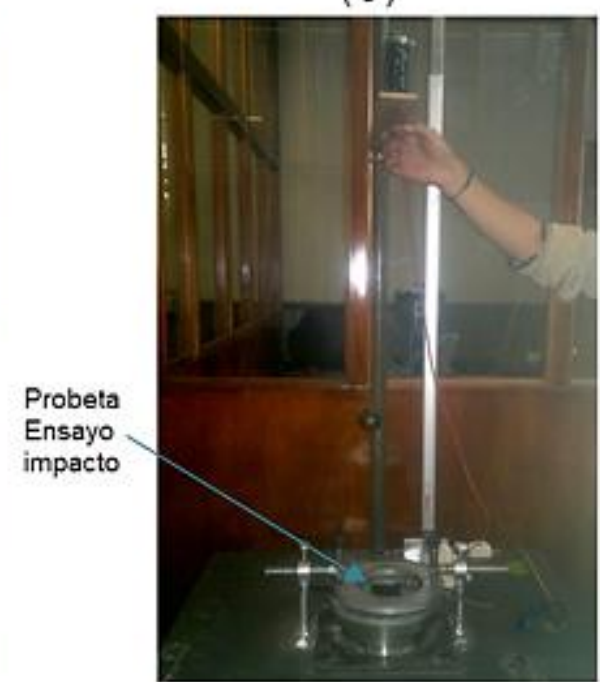

Figura 3. Equipos de ensayo y disposición de probetas: a) ensayo de tracción, b) disposición probetas para ensayo de tracción y flexión, c) ensayo de impacto - caída de dardo

Bajo directrices geométricas establecidas en (D5628 ASTM, 2010), se tomaron las probetas que obtuvieron mejores comportamientos mecánicos en los ensayos previos de tracción y flexión para someterlas a un ensayo de impacto por caída de dardo para polímeros a diferentes alturas. Se tomó como punto de partida una altura inicial de $500 \mathrm{~mm}$. Esto se efectuó progresivamente hasta 
evidenciar el colapso de las probetas, determinando consecuentemente la altura máxima de ruptura al impacto.

Posteriormente, se realizaron simulaciones de ensayos por FEM con el fin de comparar los resultados con el ensayo físico. El software ANSYS SpaceCLAIM fue empleado para obtener los ensayos de tracción. Además, se generó un modelo matemático mediante el software Explicit Dynamics de ANSYS, para determinar parámetros ingenieriles cuando la probeta sea sometida a una fuerza uniaxial y se genere un esfuerzo por tracción (Gladman, 2007). Se realizó el proceso de simulación en el módulo Autodyn y se obtuvieron los resultados esperados los cuales han sido procesados y se entregan curvas de parámetros, así como interpretación de las mismas. Se definió un tipo de malla cuadrilateral con un tamaño de elemento $2.008860 \mathrm{~mm}$ y un tamaño mínimo de malla de $0.50 \mathrm{~mm}$ con un número de nodos de 2,585 y un número tal de elementos de 2,479. El modelo matemático de Flanagan Belytschko fue utilizado para el análisis dinámico para proporcionar estabilidad a los elementos no lineales (Belytschko, Liu, Moran, \& Elkhodary, 2013; Flanagan \& Belytschko, 1981). Los resultados fueron verificados con la relación de energía de Hourglass con la energía interna generada en el modelo dando un valor de $0.75 \%$, porcentaje válido ya que según la base teórica este valor debe ser menor al 10\% (Flanagan \& Belytschko, 1981).

Finalmente; con los mejores resultados obtenidos de las pruebas preliminares de tracción, fueron comparados con los de la simulación efectuada. Seguidamente se completaron los ensayos de flexión e impacto bajo las normas prescritas para obtener los resultados de las propiedades mecánicas del material propuesto. En la última etapa del estudio, se verificó la adherencia y homogeneidad del compuesto hibrido por la técnica de microscopía de barrido SEM; sobre todo, visualizar la distribución de la fibra natural en el compuesto.

\section{Resultados y discusión}

\subsection{Ensayos preliminares material común (FV)}

La Figura 4 presenta los resultados del análisis estadístico de los esfuerzos máximos presentados por las probetas de tres de los mayores fabricantes de partes y piezas para autobuses en Ecuador. El esfuerzo máximo promedio a tracción encontrada de las probetas (resina poliéster + 3 capas de FV) obtenida mediante estratificación manual, provoca un esfuerzo máximo promedio de $71.54 \mathrm{MPa}$ y un módulo de elasticidad promedio de $4540.70 \mathrm{MPa}$.

\subsection{Ensayos de material híbrido propuesto (FV+FC)}

En la Figura 5 se presentan las curvas de esfuerzo versus deformación a tracción del material compuesto reforzado con fibra de cabuya en sus diferentes fracciones volumétricas. En dicha figura se puede apreciar que existe un aumento en el esfuerzo máximo a tracción de 78.24 MPa para la fracción volumétrica del 30\% (fibra larga), dando el mejor comportamiento mecánico respecto a la resistencia de tensión. 


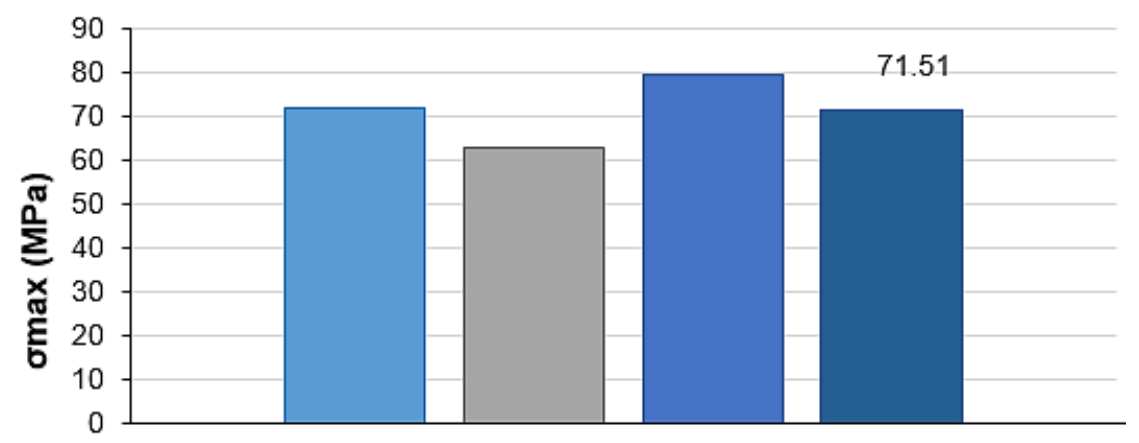

Empresas

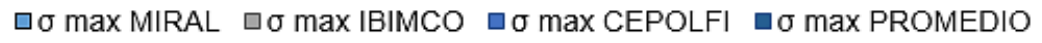

Figura 4. Ensayos preliminares de esfuerzo máximo a tracción

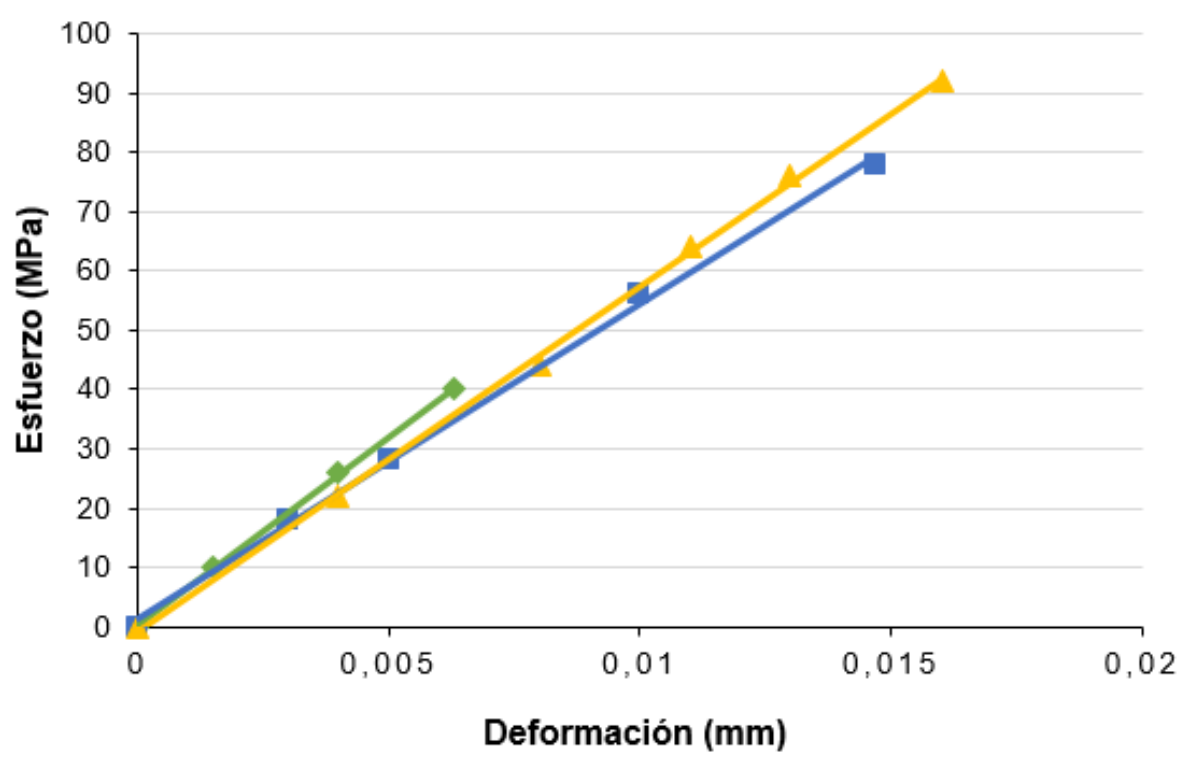

$\multimap$ Esf. Vs Def. FL $25 \% \rightarrow$ Esf. Vs Def. FL $30 \% \multimap-E s f$. Vs Def. FL $40 \%$

Figura 5. Esfuerzo versus deformación de diferentes combinaciones y fracciones volumétricas para el material híbrido $(\mathrm{FV}+\mathrm{FC}+\mathrm{FV})$

En cuanto al módulo elástico se determinó que este se incrementa a medida que la rigidez de las estratificaciones también aumentaba. Este comportamiento se observó para las probetas con mayor fracción volumétrica. Es importante resaltar que, en las probetas con tejido plano se obtuvieron los valores más altos del módulo de elasticidad respecto a las probetas de tejido continuo a lo largo de su eje principal (fibra larga). Estos resultados (Tabla 3), son concordantes y consecuentes en el comportamiento de la elongación antes de la ruptura, también evidenciado en investigaciones previas realizadas por (Bismarck, Baltazar-Y-Jiménez, \& Sarikakis, 2006; Pickering et al., 2016). Finalmente se determina que los compuestos con adición de FL 30\% de cabuya evidencian mejores resultados en cuanto al comportamiento mecánico, superando los valores esperados (figuras 5 y 6 ). Consecuentemente; estas configuraciones de fracción volumétrica son quienes pasan a la siguiente etapa del estudio para ser sometidas a pruebas de flexión e impacto; dando un buen indicio como material alternativo para aplicación automotriz. 
Tabla 3: Tabla resumen de propiedades mecánicas de las distintas estratificaciones más destacadas

\begin{tabular}{|c|c|c|c|c|c|c|c|c|c|c|c|c|}
\hline \multirow[b]{2}{*}{ Ensayo } & \multirow[b]{2}{*}{$\mathbf{e}_{\mathrm{p}}$} & \multirow[b]{2}{*}{ Dif } & \multicolumn{4}{|c|}{ TRACCIÓN } & \multicolumn{4}{|c|}{ FLEXIÓN } & \multicolumn{2}{|c|}{ IMPACTO } \\
\hline & & & $\begin{array}{c}\sigma \max \\
(\mathrm{MPa})\end{array}$ & Dif & $\begin{array}{c}E \\
(\mathrm{MPa})\end{array}$ & Dif & $\begin{array}{l}\sigma \max \\
(\mathrm{MPa})\end{array}$ & Dif & $\begin{array}{c}\text { E } \\
(\mathrm{MP} \\
\text { a) }\end{array}$ & Dif & $\begin{array}{l}\text { Rest. Máx. } \\
\text { Impacto } \\
\text { MFE=hwf [J] }\end{array}$ & Dif \\
\hline FCo $2-30 \%$ & 3.30 & 0.09 & 51.88 & 20.76 & 6258 & 1216 & 114.06 & 48.6 & 8229 & 7430 & 2.38 & 0.38 \\
\hline FL $30 \%$ & 3.01 & 0.20 & 78.24 & 5.60 & 5272 & 230 & 120.52 & 42.1 & 8053 & 7606 & 2.38 & 0.38 \\
\hline FL 40\% & 3.00 & 0.21 & 92.20 & 19.56 & 5577 & 535 & 59.28 & 103 & 1799 & 13860 & 1.54 & 0.46 \\
\hline TP $30 \%$ & 2.83 & 0.38 & 59.46 & 13.18 & 7948 & 2906 & 121.95 & 40.7 & 6751 & 8908 & 2.38 & 0.38 \\
\hline \multicolumn{13}{|c|}{ E: módulo de elasticidad } \\
\hline \multicolumn{13}{|c|}{ Ep: Espesor promedio de probetas } \\
\hline \multicolumn{13}{|c|}{ Dif: Diferencia de datos } \\
\hline
\end{tabular}

En la Figura 6, se aprecia el comportamiento del compuesto híbrido al esfuerzo máximo a la flexión en distintas fracciones volumétricas. Además de la propiedad mostrada, se determinó el módulo de flexión en la cual el compuesto combinado FL 40\% y TP 30\% ofrecieron menor comportamiento mecánico a la flexión. La estratificación FL 30\%, a pesar de evidenciar el menor esfuerzo máximo a flexión y que no está dentro de la desviación estándar esperada con el 95\% de nivel de confianza, presenta el mejor comportamiento. Basadas en estos resultados, se puede determinar que este tipo de configuración es potencialmente un material híbrido alternativo.

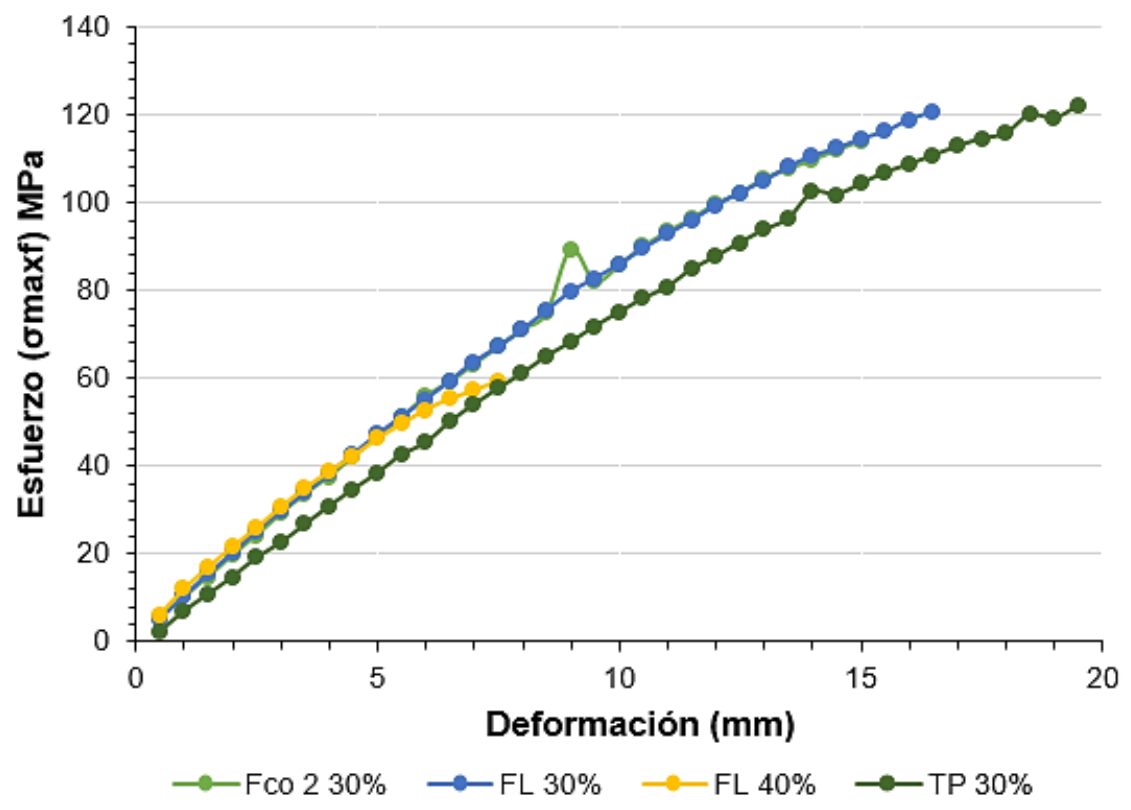

Figura 6. Esfuerzo máximo de flexión versus deformación de compuesto híbrido $(F V+F C+F V)$

Para las configuraciones FL 30\%, FCO 2 y TP 30\%; se recopilaron datos del test de impacto. Para obtener las propiedades mecánicas al impacto. Mediante configuraciones caracterizadas en función del espesor promedio, altura de lanzamiento y resistencias máximas al impacto; se observa que, las distintas fracciones volumétricas presentan comportamientos similares a la resistencia al impacto, con la particularidad que FCO 2 tiene el mayor espesor de la estratificaciones y FL-40\% el menor. Todo lo mencionado se puede observar en la Tabla 4. 
Tabla 4: Evaluación de ensayos de impacto del compuesto híbrido propuesto

\begin{tabular}{cccc}
\hline $\begin{array}{c}\text { Estratificación } \\
\text { ensayada }\end{array}$ & $\begin{array}{c}\text { Espesor } \\
\text { promedio }\end{array}$ & $\begin{array}{c}\text { Altura de lanzamiento } \\
\text { de falla }(\mathbf{m m})\end{array}$ & $\begin{array}{c}\text { Resistencia máxima al impacto } \\
\text { MFE=hwf }[\mathbf{J}]\end{array}$ \\
\hline FCo2 30\% & 3.28 & 850 & 2.38 \\
FL-30\% & 3.15 & 850 & 2.38 \\
FL- 40\% & 2.87 & 550 & 1.54 \\
\hline
\end{tabular}

\subsection{Simulación del ensayo de tracción}

La Figura 7 y la Tabla 5, ilustran los resultados del ensayo de tracción obtenidos de las pruebas experimentales de la estratificación híbrida $(F V+F C+F V)$, en ellos se muestran mejores propiedades mecánicas comparadas con la estratificación normal o comercial (FV+FC). Cabe mencionar que, en la tabla mencionada se resumen las propiedades mecánicas fundamentales de los dos tipos de estratificaciones ensayadas. Vale señalar que la convergencia de la simulación bajo radio Jacobiano fue de 1.124; lo que indica que los resultados son aceptables.

( a )

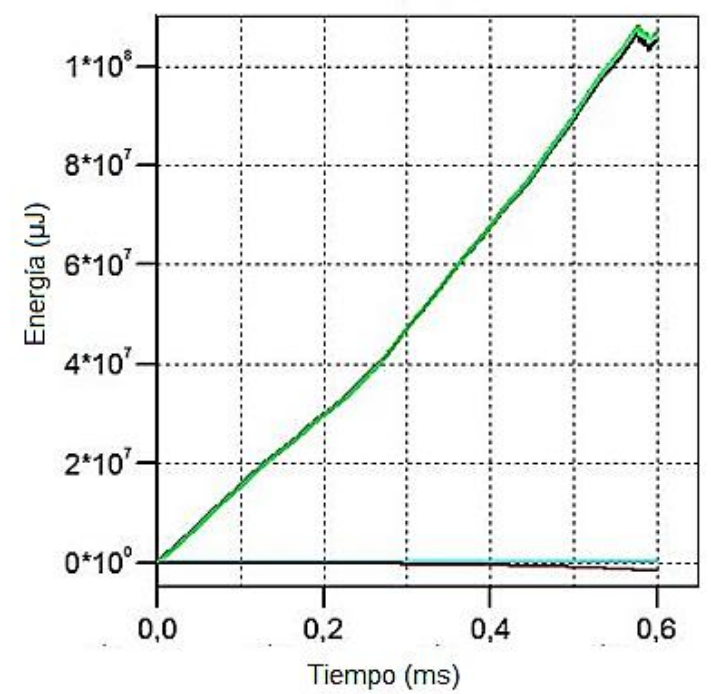

(b)

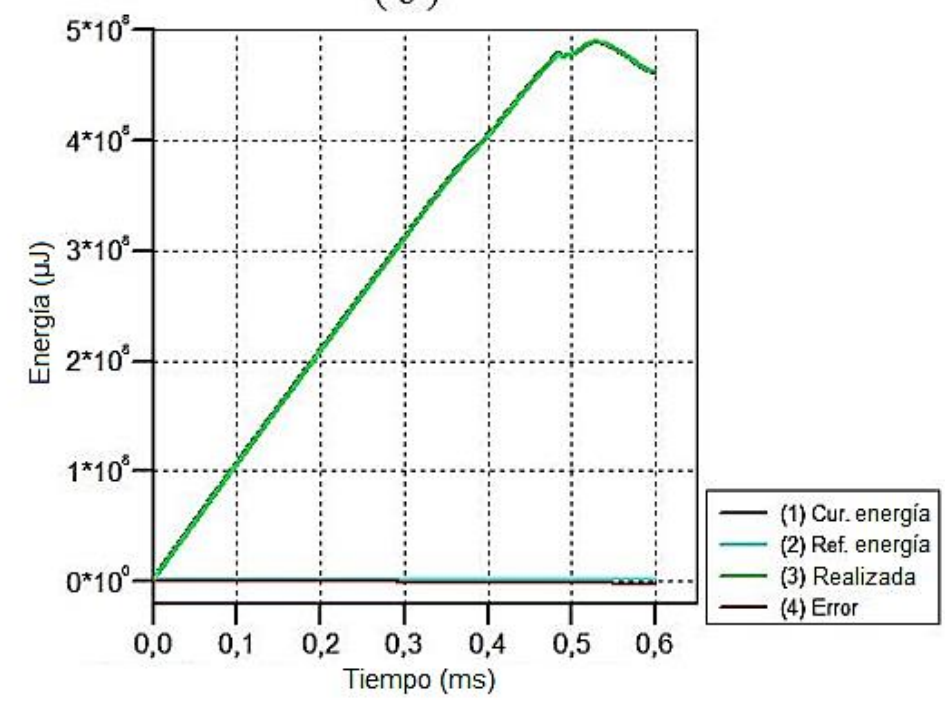

Figura 7. Curva de energía Hourglass con ANSYS: (a) estratificación (FV+FC), (b) estratificación $(\mathrm{FV}+\mathrm{FC}+\mathrm{FV})$

Tabla 5. Resultados de ensayos simulados de tracción en material compuesto en sus diferentes estratificaciones

\begin{tabular}{lcc}
\hline \multicolumn{1}{c}{ Detalle } & Mínimo [MPa] & Máximo [MPa] \\
\hline Esfuerzo máx. principal (FV+RP) & 16.41 & 71.46 \\
Esfuerzo de Von Mises (FV+RP) & 0.00 & 63.07 \\
Esfuerzo máx. principal (FV+FC+RP) & 17.97 & 78.09 \\
Esfuerzo de Von Mises (FV+FC+RP) & 17.89 & 75.24
\end{tabular}

En la Figura 8, se denota la diferencia del esfuerzo máximo en las diferentes estratificaciones simuladas por el FEM; la estratificación híbrida es un $8.5 \%$ más alta que la estratificación convencional. En el caso de la evaluación del esfuerzo de Von Mises, el compuesto híbrido es superior en un $15 \%$. Además, comparando los valores de los esfuerzos máximos obtenidos por el 
FEM y método experimental, se puede observar que hay una ligera diferencia del $0.2 \%$ entre ellos, respaldando los resultados hallados. Caso similar sucede con los desplazamientos máximos. La simulación entrega un resultado de $0.64 \mathrm{~mm}$ comparado con el ensayo real que se obtuvo de 0.7 $\mathrm{mm}$.

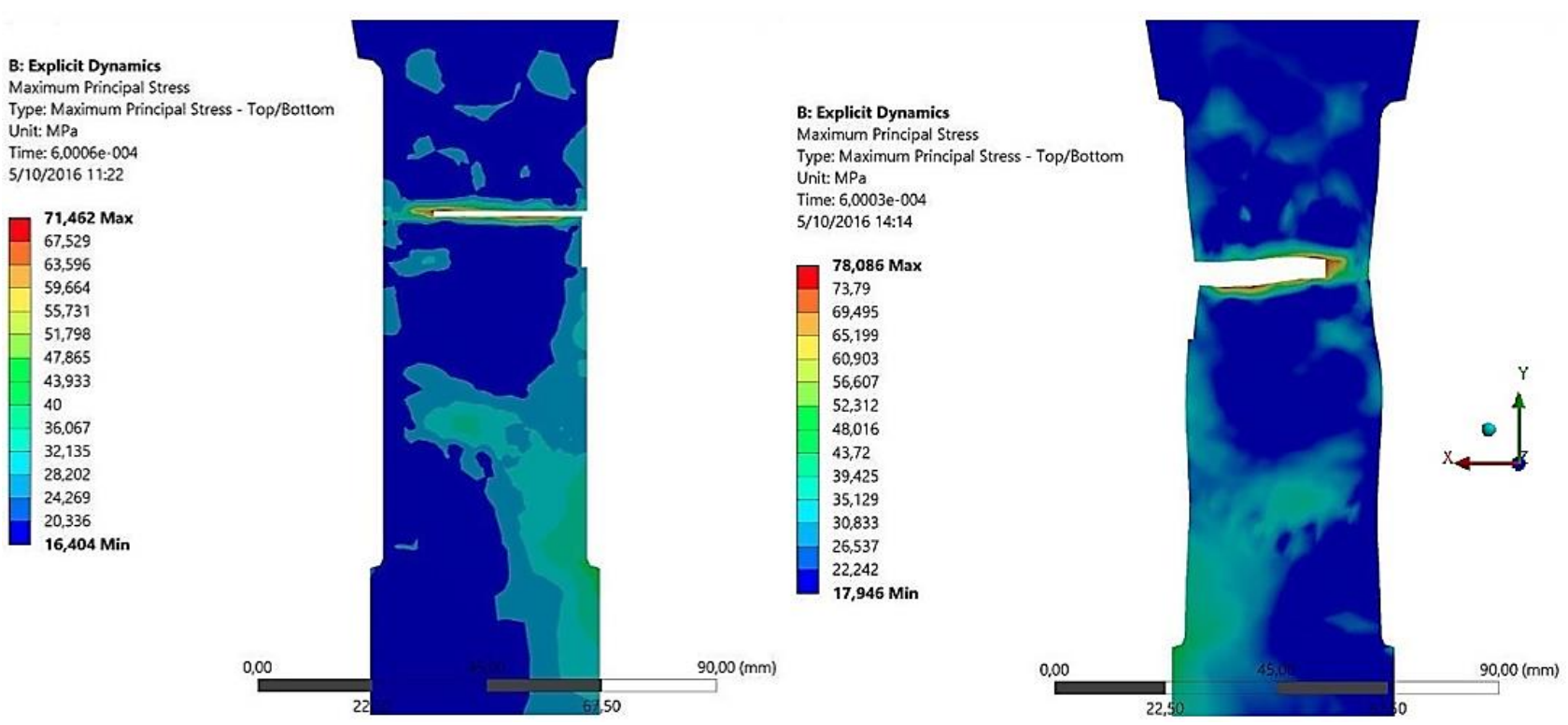

Figura 8: esfuerzo máximo de tracción (a) Estratificación $(F V+F C)$, (b) estratificación $(F V+F C+F V)$

\subsection{Propiedades del compuesto propuesto}

La Tabla 6 muestra las propiedades mecánicas del material propuesto para este análisis de resina poliéster reforzado con fibra de vidrio 375 y cabuya $(R P+F V+F C)$. La fibra de vidrio $(R P+F V)$ es un material utilizado tradicionalmente para piezas de forrado exterior e interior de autobuses; sobre todo en la manufactura ecuatoriana. Este material supera en un $10 \%$ el esfuerzo máximo comparando la estratificación tradicional con la alternativa experimentada FL $30 \%$ en las mismas condiciones.

Tabla 6. Propiedades mecánicas material FL 30\% propuesto

Propiedades físico-mecánicas

\begin{tabular}{ll}
\hline Densidad $\left(\mathrm{gr} / \mathrm{cm}^{3}\right)$ & 1.55 \\
Espesor promedio $(\mathrm{mm})$ & 3.01 \\
Longitud de fibra $(\mathrm{mm})$ & \\
Esfuerzo máximo $\sigma(\mathrm{MPa})$ & 78.24 \\
Módulo de elasticidad $\mathrm{E}(\mathrm{MPa})$ & $5,272.0$ \\
Energía de impacto $(\mathrm{J})$ & 2.38 \\
Coeficiente de Poisson & 0.3378 \\
Módulo de elasticidad transversal G(MPa) & $1,970.0$ \\
\hline
\end{tabular}




\subsection{Adherencia y homogeneidad del híbrido (matriz respecto a los refuerzos)}

El compuesto híbrido que dio mejores propiedades mecánicas (FL 30\%) y en especial a tracción, fue analizado mediante microscopía electrónica de barrido SEM. Con este procedimiento se determinó la adherencia y homogeneidad de la matriz respecto a los refuerzos, Figura 9. Se evidenció una mediana homogeneidad debido a que la cabuya proporciona poca mojabilidad (humedecimiento) respecto a la matriz (RP), por lo que es necesario un trabajo minucioso a la hora de elaborar este compuesto.

Cuando la fibra FL-30\% está alineada paralelamente a la dirección de la carga aplicada, se obtiene el mejor comportamiento mecánico, esto también se evidenció en estudios similares con fibras naturales tipo alargadas como la Cabuya (Bledzki, Mamun, Jaszkiewicz, \& Erdmann, 2010; Pickering et al., 2016). Sin embargo, es dificultoso conseguir una alineación perfecta con fibras naturales comparada con fibras sintéticas tipo continuas. Una mejoría de esto se logra en el diseño del molde y la forma del moldeado; es decir, el acomodamiento manual de la fibra natural larga en combinación con el moldeo a presión, ayuda en la obtención de mejores propiedades mecánicas.

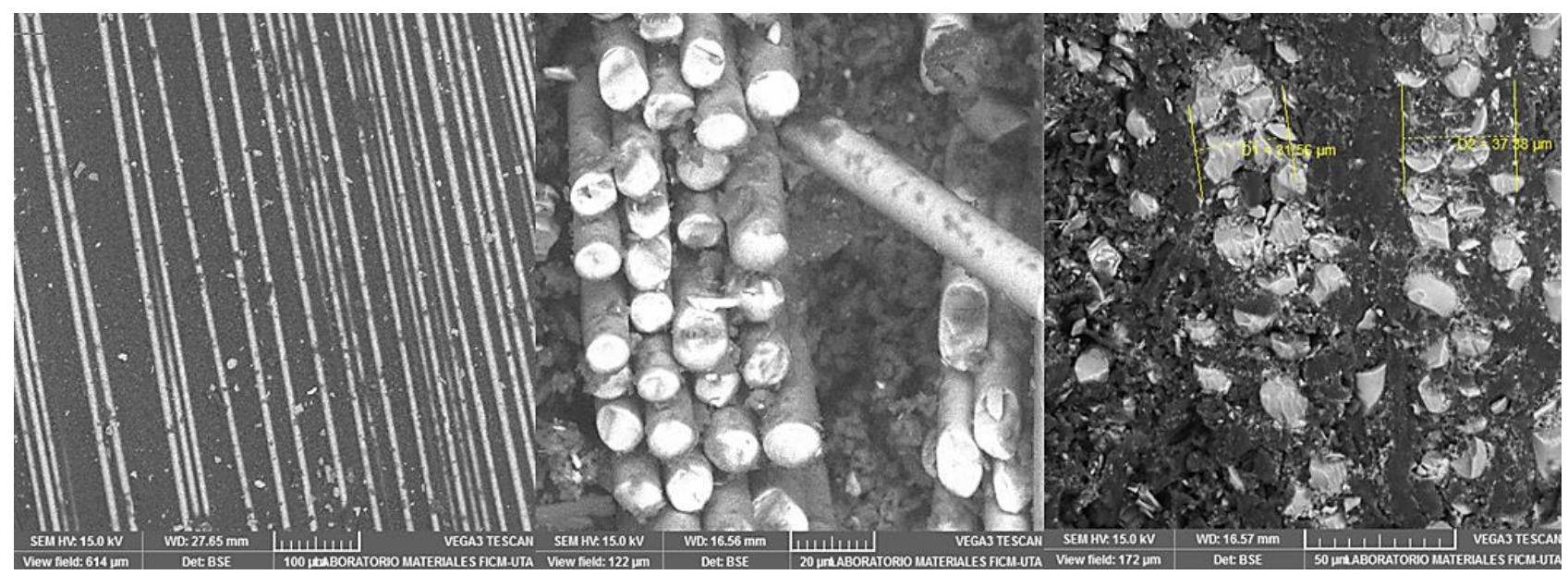

Figura 9. Fractografía del compuesto FL-30\% con mediana homogeneidad de distribución de fibras.

La estratificación a compresión por ajuste normal permitió establecer una mediana interfaz entre los dos tipos de refuerzos (FV y FC) por la baja capacidad de absorción de la cabuya que presentó cierta hermeticidad; se estableció de forma consecuente por esta razón, que siempre será necesario ubicar como refuerzo inicial una capa de FV.

\section{Conclusiones y recomendaciones}

Esta investigación buscaba determinar las propiedades mecánicas del compuesto híbrido $\mathrm{RP}+\mathrm{FV}+\mathrm{FC}$ y compararlas con las mismas propiedades en el material tradicional $\mathrm{RP}+\mathrm{FV}$; la fibra de vidrio es empleada en su gran mayoría en la producción de partes estructurales como guardachoques, frentes y respaldos de buses, techos exteriores, entre otros; y partes no estructurales como tapamáquinas, forros interiores, consolas y tableros. 
Es de gran importancia para el ambiente el desarrollo de materiales biocompuestos como el material desarrollado $\mathrm{RP}+\mathrm{FV}+\mathrm{FC}$ con características similares o mejoradas con los materiales tradicionales como la RP+FV. La incorporación de fibras de refuerzo de cabuya a las matrices poliméricas permitió un incremento de las propiedades mecánicas a tracción, flexión e impacto. Tal mejoramiento en las distintas estratificaciones dependió de las fracciones volumétricas empleadas y la configuración del refuerzo.

El compuesto híbrido de fibra larga al $30 \%$, específicamente de fracción volumétrica $70 \%$ de matriz y $30 \%$ de refuerzo con estratificación a compresión y mediante el orden de capas de refuerzo: $\mathrm{FV}+\mathrm{FC}+\mathrm{FV}$; evidenció el mejor comportamiento a las diferentes propiedades mecánicas. Este material potencialmente puede aplicarse a elementos automotrices sometidos a esfuerzos como por ejemplo guardachoques, frentes, respaldos y techos de autobuses. Además, el siguiente material en evidenciar una buena respuesta a las propiedades mencionadas es el material híbrido de fibra corta al 30 \% igualmente a estratificación a compresión e igual orden de capas del material híbrido de fibra larga al $30 \%$.

La estratificación a compresión por ajuste mecánico normal, permitió obtener una buena compactación de las fibras sintéticas y naturales (FV y FC) por la pobre capacidad de absorción evidenciada por la cabuya; se establece así que siempre será necesario ubicar como refuerzo inicial una capa de FV.

Se obtuvo una diferencia del $2 \%$ en la respuesta al esfuerzo de tracción del material híbrido resultado de los ensayos de tracción simulada por FEM con el test experimental. Lo que permite confiar en resultados de modelaciones siempre y cuando se utilice una base teórica y un método matemático concordante con las características particulares de los diferentes materiales.

La aplicación de la norma ASTM D3039-00 especificada para la caracterización a tracción de materiales poliméricos, es también aplicable para el test de los materiales híbridos propuestos, adaptándose sin problema al espesor del material, puesto que con anterioridad fue un parámetro establecido y determinado mediante el cálculo de la media aritmética, que dio como resultado 3.2 $\mathrm{mm}$.

De la bibliografía estudiada en el campo del automóvil, actualmente no utilizan materiales biocompuestos con frecuencia en aplicaciones estructurales, sino más bien en aplicaciones de bajo comportamiento mecánico como paneles en automotores y partes decorativas; dado que no se ha desarrollado tecnología que pueda manufacturar con la mayor velocidad de producción que esta industria requiere. Asímismo, en el sector del autobús hay gran potencialidad de ir remplazando los biocompuestos, ya que la manufactura de los biocompuestos con resina poliéster o epoxi es similar a la fibra de vidrio. 
Por lo mencionado, hay gran campo de exploración y estudio en la manufactura de los biocompuestos; como es, la búsqueda del mejoramiento de la adhesión, la resistencia a la humedad, mejora de propiedades mecánicas, la velocidad de producción, entre otras. Que servirán para el remplazo amigable de partes y piezas de la industria automotriz con el ambiente.

\section{Bibliografía}

ASTM, D. (2007). Standard Test Method for Flexural Properties of Polymer Matrix Composite Materials. ASTM International(West Conshohocken). doi: 10.1520/D7264_D7264M-07

ASTM, D. (2008). Standard test method for tensile properties of polymer matrix composite materials. ASTM International, West Conshohocken, PA,. doi: 10.1520/D0638-08

ASTM, D. (2010). Standard Test Method for Impact Resistance of Flat, Rigid Plastic Specimens by Means of a Falling Dart (Tup or Falling Mass). ASTM International, West Conshohocken, PA,. doi: 10.1520/D5628-10

Belytschko, T., Liu, W. K., Moran, B., \& Elkhodary, K. (2013). Nonlinear finite elements for continua and structures: John wiley \& sons.

Bismarck, A., Baltazar-Y-Jiménez, A., \& Sarikakis, K. (2006). Green composites as panacea? Socioeconomic aspects of green materials. Environment, Development and Sustainability, 8(3), 445463.

Bledzki, A. K., Mamun, A. A., Jaszkiewicz, A., \& Erdmann, K. (2010). Polypropylene composites with enzyme modified abaca fibre. Composites Science and Technology, 70(5), 854-860.

Cao, Y., Shibata, S., \& Fukumoto, I. (2006). Mechanical properties of biodegradable composites reinforced with bagasse fibre before and after alkali treatments. Composites Part A: Applied Science and Manufacturing, 37(3), 423-429.

Casanova, L. (2010). La inversión extranjera directa en América Latina y las multinacionales emergentes latinoamericanas. Boletín Elcano(128), 7.

Chiaberge, M. (2011). New Trends and Developments in Automotive Industry.

Chung, D. D. (2010). Composite materials: science and applications: Springer Science \& Business Media.

Dieu, T. V., Phai, L. T., Ngoc, P. M., Tung, N. H., \& Le Phuong, T. (2004). Study on preparation of polymer composites based on polypropylene reinforced by jute fibers. JSME International Journal Series A Solid Mechanics and Material Engineering, 47(4), 547-550. 
Flanagan, D., \& Belytschko, T. (1981). A uniform strain hexahedron and quadrilateral with orthogonal hourglass control. International journal for numerical methods in engineering, 17(5), 679-706.

Fowler, P. A., Hughes, J. M., \& Elias, R. M. (2006). Biocomposites: technology, environmental credentials and market forces. Journal of the Science of Food and Agriculture, 86(12), 17811789 .

Gladman, B. (2007). LS-Dyna Keyword Users' Manual. Livermore Software Corporation California.

Hallal, A., Elmarakbi, A., Shaito, A., \& El-Hage, H. (2013). Overview of Composite Materials and their Automotive Applications. Advanced Composite Materials for Automotive Applications: Structural Integrity and Crashworthiness, 1-28.

Hull, D. (2003). Materiales compuestos (Reverté Ed. Primera ed.). Barcelona.

McWilliams, A. (2015). Lightweight Materials in Transportation Advanced Materials: BBC Research.

Pickering, K. L., Efendy, M. A., \& Le, T. M. (2016). A review of recent developments in natural fibre composites and their mechanical performance. Composites Part A: Applied Science and Manufacturing, 83, 98-112.

Shaharuddin, S., \& Matthews, Z. (1994). FL and Rawlings, RD, Composite Materials: Engineering and Science: Chapman \& Hall, London.

Shibata, S., Cao, Y., \& Fukumoto, I. (2005). Press forming of short natural fiber-reinforced biodegradable resin: Effects of fiber volume and length on flexural properties. Polymer testing, 24(8), 1005-1011.

Smith, W. F., Hashemi, J., Cázares, G. N., Caver, P. A. G., Avilés, L. C., \& Velasco, J. A. B. (2006). Fundamentos de la ciencia e ingeniería de materiales: McGraw-Hill. 